\begin{tabular}{|c|c|}
\hline Title & Facilitation of temporal prediction by electrical stimulation to the primate cerebellar nuclei \\
\hline Author(s) & Uematsu, A kiko; Ohmae, Shogo; Tanaka, Masaki \\
\hline Citation & $\begin{array}{l}\text { Neuroscience, 346, 190-196 } \\
\text { https://doi.org/10.1016/.neuroscience.2017.01.023 }\end{array}$ \\
\hline Issue Date & $2017-03-27$ \\
\hline Doc URL & http:/hdl .handle.net/2115/68588 \\
\hline Rights & $\begin{array}{l}\text { ( ) 2017. This manuscript version is made available under the CC-BY-NC-ND } 4.0 \text { license } \\
\text { http://creativecommons.org/icenses/by-nc-nd/4.0/ }\end{array}$ \\
\hline Rights(URL) & http://creativecommons.org/icenses/by-nc-nd/4.0/ \\
\hline Type & article (author version) \\
\hline File Information & Neuroscience346_190.pdf \\
\hline
\end{tabular}

Instructions for use 


\title{
Facilitation of temporal prediction by electrical stimulation to the primate cerebellar nuclei
}

\author{
Akiko Uematsu $^{\mathrm{a}}$, Shogo Ohmae ${ }^{\mathrm{a}, \mathrm{b}}$ and Masaki Tanaka ${ }^{\mathrm{a}}$ \\ a Department of Physiology, Hokkaido University School of Medicine, Sapporo 060-8638, \\ Japan \\ ${ }^{\mathrm{b}}$ Department of Neuroscience, Baylor College of Medicine, Houston, TX 77030, USA
}

Text pages: 16

Figures: 6

Table: 0

Abstract: 215 words

Correspondence to:

Dr. Masaki Tanaka

Department of Physiology

Hokkaido University School of Medicine

North 15, West 7, Sapporo 060-8638, Japan

Tel: +81 11-706-5039, Fax: +81 11-706-5041

E-mail:masaki@med.hokudai.ac.jp 


\section{Abstract}

The cerebellum is known to be involved in temporal information processing. However, the underlying neuronal mechanisms remain unclear. In our previous study, monkeys were trained to make a saccade in response to a single omission of periodically presented visual stimuli. To detect stimulus omission, animals had to predict the timing of each next stimulus. During this task, neurons in the cerebellar dentate nucleus exhibited a transient decrement of activity followed by a gradual increase in firing rate that peaked around the time of the next stimulus (Ohmae et al., 2013). In the present study, to address how these two components of neuronal activity contributed to omission detection, we applied electrical microstimulation to the recording site at different timing during the task. We found that electrical stimulation just before the stimulus omission shortened the latencies of both contraversive and ipsiversive saccades. Because the changes in saccade latency non-linearly depended on the timing of stimulation in each inter-stimulus interval, and electrical stimulation just before the early stimulus in the sequence failed to evoke saccades, the neuronal activity in the dentate nucleus might regulate temporal prediction rather than facilitating saccade execution. Our results support the hypothesis that the firing modulation in each inter-stimulus interval in the dentate nucleus represents neuronal code for the temporal prediction of next stimulus.

\section{Abbreviations}

FP, fixation point; MRI, magnetic resonance images; SOA, stimulus onset asynchrony

\section{Key words}

temporal processing; electrical stimulation; dentate nucleus; cerebellum; primate 


\section{Introduction}

Temporal prediction is essential for both motor control and cognition. Although the involvement of the cerebellum in motor control has long been recognized, recent evidence shows that the cerebellum also plays a role in temporal prediction of sensory events (Coull and Nobre, 1998; O’Reilly et al., 2008; Roth et al., 2013; Avanzino et al., 2015). While much is known about the underlying neuronal mechanism of the cerebellar control of movements (for reviews, see Thach et al., 1992; Ito 2011; Manto et al., 2012), how neuronal signals in the cerebellum regulate temporal prediction of external events remains elusive.

We recently found that neurons in the cerebellar dentate nucleus exhibited characteristic firing modulation for periodically presented audiovisual stimuli (Ohmae et al., 2013). In our "missing oddball" paradigm, monkeys predicted the timing of each next stimulus in the sequence so that they generated a saccade in response to a single omission of repetitive stimuli. Neurons in the posterior part of the dentate nucleus exhibited a transient decrement of firing rate for each stimulus presentation, which was followed by a gradual increase in firing rate. The degree of the initial decrement of activity was proportional to the inter-stimulus interval (i.e., the time from the preceding stimulus) and the neuronal firing rate reached a peak around the time of the next stimulus. Because pharmacological inactivation of the recording sites delayed the detection of stimulus omission but not the detection of stimulus change, the temporally-specific signals found in the cerebellum likely regulated the temporal prediction of the periodic stimuli that was necessary for omission detection (Ohmae et al., 2013; Ohmae and Tanaka, 2016).

However, it remains uncertain how the signals in the cerebellar dentate nucleus regulate the detection of stimulus omission. Although the previous inactivation results clearly proved the causality, we were unable to associate patterns of neuronal activity with behavioral outcomes. Specifically, it was unclear whether the transient decrement or the gradual increment of firing rate played a role, or, alternatively, if neuronal modulation reflected the preparation of specific movements. Furthermore, because the gradual elevation of sensory gain in each trial and the firing modulation in each inter-stimulus interval had different time courses, they might play different roles in controlling the behavioral performance in the task. To address these issues, we applied electrical microstimulation to the recording sites in the dentate nucleus at various timing during the missing oddball task. Some of the present results have been reported in abstract form (Uematsu and Tanaka, 2015). 


\section{Experimental procedures}

\section{Animal preparation}

Four Japanese monkeys (Macaca fuscata, 6-9 kg, two females, monkeys H, A, I and Hk) were used. All experimental protocols were evaluated and approved by the Hokkaido University Animal Care and Use Committee. Much of the experimental procedure was the same as those described previously (Tanaka, 2005). Monkeys were initially trained to sit in a primate chair. Animals were then implanted with a pair of head holders and an eye coil in two separate surgeries. All of the surgeries were executed under general isoflurane anesthesia using sterile procedures. Analgesics were also administrated during each surgery and several days later. After full recovery from the surgery, the animals were trained in the oculomotor version of the missing oddball detection task (Ohmae et al., 2013, see the following section). During the training and experimental sessions, animals' heads were secured to the primate chair in a darkened booth. Horizontal and vertical eye position were recorded using the search coil technique (MEL-25, Enzanshi Kogyo). After sufficient training on the task, a recording chamber was placed to enable vertical electrode penetration, targeted at the cerebellar dentate nucleus. The chamber location relative to the dentate nucleus was verified using magnetic resonance imaging (MRI) after the surgery.

\section{Visual stimuli and behavioral task}

Experiments were controlled by a Windows-based stimulus presentation and data acquisition system (TEMPO, Reflective Computing). Visual stimuli were presented on a 24-inch cathode ray tube monitor (refresh rate, $60 \mathrm{~Hz}$ ) positioned $38 \mathrm{~cm}$ from the eyes. The monitor subtended $64 \times 44^{\circ}$ of visual angle.

In the missing oddball task (Fig. 1A; Ohmae et al., 2013), a fixation point (FP, red $0.5^{\circ}$ square) was initially presented at the center of the screen. After the animals gazed at the FP, a saccade target (gray $0.5^{\circ}$ or $1.0^{\circ}$ square) appeared either $16^{\circ}$ left or right of the FP. A brief visual stimulus (white unfilled $2^{\circ}$ square, $35 \mathrm{~ms}$ or 2 video frames in duration) surrounding the FP was presented repeatedly at a fixed stimulus onset asynchrony (SOA) of either 150, 400, or $600 \mathrm{~ms}$. An auditory tone $(1500 \mathrm{~Hz}$ square wave, $35 \mathrm{~ms})$ was also presented synchronously with the visual stimulus in all but six stimulation experiments in monkey H. After a random 3600-5200 ms period (2000-4800 ms for recording experiments), one stimulus in the series was omitted (missing oddball). The animals were required to make a saccade to the visible target in response to the stimulus omission within $600 \mathrm{~ms}$. Successful 
performance was rewarded with drops of liquid reward. To compare the effects of electrical stimulation, we also presented the visually-guided saccade task, in which a central fixation point (yellow $1.0^{\circ}$ square) was replaced by a saccade target that was located $16^{\circ}$ horizontally. Monkeys were rewarded for saccades within 700 ms following the target onset.

\section{Physiological procedures}

Before each stimulation experiment, we recorded from neurons in the dentate nucleus of the cerebellum using single tungsten electrodes (Alpha Omega Engineering). The properties of the task-related neurons have been described previously in detail (Ohmae et al., 2013). The location of each electrode penetration was adjusted using the grid system (Crist Instruments) attached to the recording chamber. During experiments, the electrode was inserted into the brain through a 23-gauge stainless steel tube and was advanced remotely using a micromanipulator (MO-97S, Narishige). Signals obtained from the electrodes were amplified, filtered, and analyzed online using a spike sorter with template-matching algorithm (MSD or ASD, Alpha Omega Engineering) to isolate single neurons.

We applied electrical stimulation to the recording sites at various timing during the task. Electrical stimulation was a train of 0.2-ms biphasic pulses delivered through the recording electrode. The frequency of stimulation pulses was either 200 or $333 \mathrm{~Hz}$, and the duration was 100 or 200 ms. The intensity of stimulation current was monitored by measuring the voltage across a serially connected $1-\mathrm{k} \Omega$ resistor, and was adjusted within a range of 80 $100 \mu \mathrm{A}$. According to the previous study (Holdefer et al., 2000), this current intensity was effective to elicit neuronal response in the cerebral cortex. Because electrical stimulation did not evoke any obvious movement and exhibited only a small change in saccade latency (see Results), we used the same stimulation parameters throughout the experiments. Once we encountered task-related neuronal activity or reached the region in which we had previously recorded the task-related neurons, electrical stimulation was applied 100-500 ms before stimulus omission or a repetitive visual stimulus. Details of stimulation parameters are reported in the relevant text in the Results and in the figure legends.

\section{Data acquisition and analysis}

Data were digitized and sampled at $1 \mathrm{kHz}$, and were saved in files during experiments. Offline analysis was performed using Matlab (Mathworks). Reaction time was defined as the time from stimulus omission to saccade initiation. If electrical stimulation at a given site 
altered saccade latency in any condition (Wilcoxon rank-sum test, $p<0.05$ ), the data were included in further analysis.

Locations of electrical microstimulation in monkeys $\mathrm{H}$ and $\mathrm{I}$ were verified histologically based on the stereotaxic coordinates and landmark pins (Fig. 1B). After the termination of all experiments, monkey I was deeply anesthetized with pentobarbital sodium (i.p., $60 \mathrm{mg} / \mathrm{kg}$ ) and was perfused transcardially with $0.1 \mathrm{mM}$ phosphate buffer followed by 3.5\% formalin. Monkey $\mathrm{H}$ died unexpectedly from acute gastrointestinal dilation. Immediately after the death, we inserted the landmark pins through the recording cylinder and perfused the animal through the carotid artery. After fixation with 3.5\% formalin, the brain of each monkey was removed, blocked and equilibrated with $30 \%$ sucrose. We used a freezing microtome to cut coronal sections in $100-\mu \mathrm{m}$ thickness and the histological sections were stained with cresyl violet. We also verified the locations of electrical stimulation based on MRI in the other two monkeys. While the stimulation sites were consistently located in the posterior part of the dentate nucleus in both animals, only the data from monkey A are shown in Figure 1B.

\section{Results}

\section{Electrical microstimulation promoted the detection of stimulus omission}

To locate the stimulation sites, we initially recorded from neurons in the cerebellar dentate nucleus that exhibited firing modulation for the repetitive stimuli during the oddball task (Fig. 2A). Consistent with the previous study (Ohmae et al., 2013), the magnitude of firing modulation after stimulus repetition was proportional to the SOA. For long SOAs, the firing rate peaked around the time of stimulus presentation, which appeared to represent the neural code for the temporal prediction of each next stimulus. To clarify the causal role of these signals, we applied electrical microstimulation to the recording site. At a representative site shown in Figure 2B, saccades occurred earlier in all SOA conditions when electrical stimulation was delivered 100 ms before the stimulus omission (Wilcoxon's rank sum test, $p$ $<0.05)$.

We compared reaction time between the trials with and without microstimulation to 53 sites in four dentate nuclei in four monkeys (Fig. 3). Electrical stimulation was delivered during 100 ms before the stimulus omission in three animals except for six stimulation sites in monkey I and three sites in monkey H, in which stimulation was delivered during 200 ms. For individual experiments, electrical stimulation in 41 (77\%) and 38 (72\%) sites significantly 
altered the latencies of contraversive and ipsiversive saccades, respectively (Wilcoxon's rank sum test, $p<0.05)$. In the population, a three-way ANOVA revealed a significant effect of electrical stimulation $\left(F_{(1,34)}=10.50, p<0.01\right)$ but showed no significant effect of SOA $\left(F_{(2,}\right.$ $34)=2.10, p=0.14)$ or saccade direction $\left(F_{(1,34)}=1.14, p=0.29\right)$. No significant interactions were found between the factors (stimulation $\times \mathrm{SOA}, F_{(2,34)}=0.04, p=0.96$; stimulation $\times$ direction, $F_{(1,34)}=0.001, p=0.97$; direction $\times$ SOA, $\left.F_{(2,34)}=0.06, p=0.94\right)$. Thus, the results indicated that electrical microstimulation promoted the detection of stimulus omission irrespective of SOA and saccade direction.

To exclude the possibility that electrical stimulation facilitated saccade initiation rather than the detection of stimulus omission, we also compared the stimulation effects between the visually-guided saccade trials and the missing oddball trials. When we applied electrical stimulation just before the target onset $(100 \mathrm{~ms})$ in the visually-guided saccade task, reaction time significantly altered only one of 20 cases in 10 experiments (Wilcoxon rank-sum test, $p<0.05)$ and the changes in latency averaged $-1.4 \pm 10.8 \mathrm{~ms}(\mathrm{SD}, n=20$, 10 experiments in both directions). On the other hand, the same stimulation pulses delivered during the missing oddball task altered saccade latency by $-59.2 \pm 47.3 \mathrm{~ms}(\mathrm{SD}, n=20)$, which were statistically greater than the amount in the visually-guided saccade task (one tailed paired t-test, $p<0.01)$.

\section{Effects of stimulation timing}

Given that neurons in the cerebellar dentate nucleus modulated the firing rate during each inter-stimulus interval (Figs. 2A and 4D), the effects of electrical microstimulation might depend on its timing. To test this possibility, we applied electrical stimulation (100 ms) at different timing (100-500 ms) before stimulus omission in trials with a 400-ms SOA. Figure 4A depicts the distributions of reaction time for individual trials in 42 experiments in two monkeys (A and Hk). Inverted red triangles indicate the median for each condition, and shows that saccade latency was gradually altered as the timing of electrical stimulation advanced. Although electrical stimulation well before the stimulus omission sometimes evoked erroneously early saccades, stimulation effects were also evident for saccades generated following the stimulus omission, as compared the distributions of saccade latencies between the trials with and without electrical stimulation (Fig. 4A).

Figure 4B summarizes the means of median latency for 42 experimental sessions with different stimulation timing. The data revealed that while the timing of electrical 
stimulation advanced in steps of $100 \mathrm{~ms}$, the saccade latency did not reduce steadily at a constant rate. This was evident when we plot the changes in latency for each step of stimulation timing (Fig. 4C). These results indicate that electrical stimulation did not evoke saccades with a fixed delay, and that the effects of microstimulation were modified by intrinsic neuronal signals during the task.

\section{Absence of stimulation effects during the early stages of the trial}

Neurons in the cerebellar dentate nucleus exhibited the gradual increase of firing modulation as the repetition progressed (Fig. 2A; Ohmae et al., 2013). To assess the relationship between this feature of neuronal activity and the effects of electrical stimulation, we also applied stimulation just before the early (2nd-8th) stimuli in the series of repetitive stimuli with a 400-ms SOA. Figure 5 shows the proportion of trials in which saccades directed toward the target were generated within $600 \mathrm{~ms}$ following electrical stimulation. Although saccades were generated in approximately $10 \%$ of trials when stimulation was delivered just before the 10th stimulus, stimulation applied before the early stimuli failed to evoke saccades in most trials. Furthermore, in trials without stimulation-evoked saccades, the reaction time following the regular stimulus omission did not differ across trials with different stimulation timing (one-way ANOVA, $F_{(5,108)}=0.82, p=0.54$ ). These results indicated that the enhancement of firing modulation was necessary for generating saccades in response to electrical stimulation, and that the perturbation of neuronal signals during the early stages of the trial did not affect the detection of stimulus omission.

\section{Discussion}

We found that electrical stimulation to the cerebellar dentate nucleus facilitated the detection of stimulus omission in the missing oddball task. Although our previous study demonstrated that inactivation of the dentate nucleus delayed the detection of stimulus omission (Ohmae et al. 2013), it was unclear which components of neuronal modulation played a crucial role. The present results were consistent with the hypothesis that the modulation of firing rate during each inter-stimulus interval represented the prediction of each stimulus timing.

\section{Relevance of signals in the cerebellar dentate nucleus to temporal prediction}

Because a subset of neurons in the cerebellar dentate nucleus project to the superior colliculus (May et al., 1990) and the frontal eye field via the thalamus (Lynch et al. 1994), signals in the 
dentate nucleus could play a role in triggering saccades. Previous studies showed that electrical stimulation applied to the dentate nucleus evoked ipsiversive saccades with a short latency ranging from 12-26 ms (Ron and Robinson, 1973), and that neurons in the lateral cerebellar cortex projecting to the dentate nucleus exhibited a brief burst of activity associated with saccades (Mano et al., 1991). However, the present results revealed that electrical stimulation delivered to our recording sites shortened saccade latencies in both directions, and that the latencies still remained $>200$ ms (Figs. 3 and 4A). These results suggest that neurons in our stimulation sites were unlikely to transmit immediate saccade drive.

Nevertheless, because the animals made a saccade in response to the stimulus omission in our behavioral paradigm, neuronal activity in the dentate nucleus might reflect saccade preparation. Indeed, the previous studies have shown that neurons in the caudal part of the dentate nucleus exhibit a gradual buildup of activity prior to saccades (Ashmore and Sommer, 2013). In our results, however, electrical stimulation shortened reaction time during the missing oddball task, but failed to alter latency of visually-guided saccades, suggesting that electrical stimulation might facilitate oddball detection rather than saccade preparation.

The present results and our previous studies using the missing oddball paradigm suggest a role of the dentate nucleus in timing. For example, neuronal firing rate did not modulate significantly for stimulus sequence with SOAs < 200 ms (Fig. 2; Ohmae et al., 2013), suggesting that the neuronal modulation may not be solely attributed to saccade preparation. Because the prediction of each stimulus timing is only possible for SOAs longer than several hundred milliseconds in humans (Ohmae and Tanaka, 2016), the neuronal modulation for longer SOAs is likely to be related to the prediction of stimulus timing. In addition, the previous study demonstrated that inactivation of the dentate nucleus prolonged saccade latency for stimulus omission but not for the changes in stimulus color or size (Ohmae et al., 2013), suggesting a role of this brain region in temporal processing. Furthermore, in the current study, electrical stimulation applied to the recording sites facilitated saccade initiation irrespective of its direction (Fig. 3), indicating that stimulation promoted the detection of stimulus omission rather than the preparation of specific saccades.

The ventral portion of the cerebellar dentate nucleus sends signals to the association areas in the frontal and parietal cortices (Strick et al., 2009; Ramnani 2012), which contain neurons predicting event timing (Leon and Shadlen 2003; Maimon and Assad 2006; Oshio et al., 2008; Merchant et al., 2011; Yumoto et al., 2011; Schneider and Ghose 2012; Jazayeri and Shadlen, 2015). These findings indicate that electrical stimulation may advance the prediction 
of stimulus occurrence, causing early responses. In fact, when we delivered electrical stimulation well before the stimulus omission, monkeys sometimes generated early saccades (Fig. 4A). In future studies, motor preparation and temporal prediction could be dissociated by recording neuronal activity during the tasks requiring movements with different body parts to report stimulus omission.

\section{Possible neuronal mechanisms representing temporal information}

Neuronal activity in the dentate nucleus exhibited two conspicuous features (Ohmae et al., 2013). First, the firing modulation for each repetitive stimulus was absent for the first few stimuli in the sequence but increased gradually as the repetition progressed (Fig. 2A). Second, the magnitude of transient suppression of neuronal firing rate for each repetitive stimulus was proportional to the inter-stimulus interval. This transient suppression was immediately followed by a gradual increase of firing rate that peaked around the time of the next stimulus. These features may reflect temporal prediction of external events with two different timescales (Fig. 6A).

The time course of the buildup of response gain during the stimulus repetition well predicted the timing of stimulus omission that occurred between 3600-5200 ms following the first stimulus in the sequence. Behavioral analysis in our previous study revealed that the detection of stimulus omission was worse for trials with earlier oddball occurrence (Ohmae et al., 2013), likely because the animals were not ready to detect stimulus omission during the early phase of stimulus repetition. We speculate that the gradual elevation of response gain for each stimulus was related to the animals' expectation of oddball occurrence, which might be necessary to trigger saccades in response to stimulus omission. Consistent with this hypothesis, the current results revealed that electrical stimulation applied before the early stimulus in the sequence failed to evoke saccades (Fig. 5). Conversely, electrical stimulation applied during the trials with a 150-ms SOA promoted saccades (Figs. 2B and 3), even though neuronal modulation was only small in those trials. These results suggest that both the expectation of stimulus omission in each trial and the high-gain firing modulation related to the prediction of next stimulus timing were needed to detect stimulus omission (Fig. 6B). Although the expectation of stimulus omission appears to regulate sensory gain in the cerebellum, it might be generated outside of the cerebellum.

The timing of the peak of the periodic firing modulation in the cerebellum may be relevant to the accurate prediction of the timing of each next stimulus (Fig. 6A). Since the 
magnitude of the suppressive response to each stimulus was proportional to the time from the preceding stimulus, the time course of recovery from transient suppression depended on the inter-stimulus interval (Fig. 2A). We previously found that the magnitude of recovery was correlated with the trial-by-trial variation of saccade latency in the missing oddball trials (Ohmae et al., 2013). In the present study, electrical stimulation just before stimulus omission shortened saccade latencies, but those following stimulus presentation exhibited much weaker effects than those expected from the stimulation timing. For example, Figure 4C plots the changes in saccade latency as electrical stimulation advanced in a step of $100 \mathrm{~ms}$, showing that the decrement of latency was saturated around the time of the last stimulus presentation. This might be relevant to the fact that neuronal activity was suppressed just after the occurrence of each repetitive stimulus (Fig. 4D). These observations support the notion that each stimulus presentation resets the signals for temporal prediction that gradually increase over time until the time of the next stimulus. The relatively long-lasting stimulation effects (Figs. 2 and 4) suggest that these cerebellar output signals must be further processed to generate the prediction error signals for stimulus omission that ultimately trigger saccades. Electrical stimulation may advance the predicted timing of stimulus occurrence, causing early and sometimes erroneous detection of stimulus absence (Fig. 4A). The early saccades before the stimulus omission often had similar latencies following electrical stimulation (e.g., Fig. 4A, bottom panel), likely because the strong cerebellar outputs might be immediately converted into the prediction error signals triggering saccades. Nevertheless, the effects of electrical stimulation were still evident for saccades with longer latencies, as compared the latency distributions between the trials with and without electrical stimulation.

In the present study, electrical stimulation shortened saccade latency in trials with both 150- and 400-ms SOAs, while we previously found that inactivation of the recording sites delayed saccades only for trials with SOAs > 400 ms (Ohmae et al., 2013). However, these seemingly contradictory results are in accord with the hypothesis that the brain automatically selects two alternative mechanisms of omission detection depending on the SOA (Ohmae and Tanaka, 2016); omission detection for shorter SOAs (e.g., < $250 \mathrm{~ms}$ ) may rely on temporal grouping of discrete stimuli that likely takes place outside of the cerebellum (Fig. 6B). In contrast, omission detection for longer SOAs may require temporal prediction, in which the cerebellum may participate. Assuming that the brain concurrently monitors the signals arising from two different systems, which are related to either a brief termination of sensory stream or the prediction error for stimulus omission, the earliest available signal 
might trigger saccades (Fig. 6B). Although the prediction signals in the cerebellum are absent for shorter SOAs in natural situations, electrical stimulation applied to the dentate nucleus may artificially generate signals for omission detection.

We do not claim that the cerebellum does not have a capacity of timing control for short time intervals. In fact, we previously found that a subset of neurons in the cerebellar dentate nucleus exhibited a transient activity following the stimulus omission in trials with short SOAs (Fig. 5 of Ohmae et al., 2013). Although the previous inactivation experiments suggested that these signals played virtually no role in the detection of stimulus omission with short SOAs, the same signals might play some role in other behavioral conditions. Furthermore, given that the cerebellum controls movements with an accuracy of tens of milliseconds (Thier et al., 2000; Fuchs et al., 2010), the other part of the cerebellum might regulate sensory prediction for short time intervals.

In summary, the present results suggest that neuronal activity in the dentate nucleus may reflect the temporal prediction of stimulus appearance. These signals may be further integrated in the downstream structures to produce prediction error signals for the absence of a regular stimulus (Fig. 6B). In future studies, investigation of the roles of the thalamus, cerebral cortex and basal ganglia may reveal how the temporal prediction signals in the cerebellum regulate temporal perception and timed behavior.

\section{Acknowledgements}

The authors thank T. Mori and A. Hironaka for their technical assistance, M. Suzuki for her administrative help, and M. Takei and Y. Hirata for manufacturing some equipment. This work was supported by grants from the Ministry of Education, Culture, Sports, Science and Technology of Japan, the Ministry of Health, Labour and Welfare of Japan and the Takeda Science Foundation. The authors declare no competing commercial interest. 


\section{References}

Ashmore RC, Sommer MA (2013) Delay activity of saccade-related neurons in the caudal dentate nucleus of the macaque cerebellum. J Neurophysiol 109:2129-2144.

Avanzino L, Bove M, Pelosin E, Ogliastro C, Lagravinese G, Martino D (2015) The cerebellum predicts the temporal consequences of observed motor acts. PLoS One 10:114.

Coull JT, Nobre AC (1998) Where and when to pay attention: the neural systems for directing attention to spatial locations and to time intervals as revealed by both PET and fMRI. J Neurosci 18:7426-7435.

Fuchs AF, Brettler S, Ling L (2010) Head-free gaze shifts provide further insights into the role of the medial cerebellum in the control of primate saccadic eye movements. $\mathrm{J}$ Neurophysiol 103:2158-2173.

Holdefer RN, Miller LE, Chen LL, Houk JC (2000) Functional connectivity between cerebellum and primary motor cortex in the awake monkey. J Neurophysiol 84:585-590.

Ito M (2011) The cerebellum: brain for an implicit self. New York: FT Press Science.

Jazayeri M, Shadlen MN (2015) A Neural Mechanism for Sensing and Reproducing a Time Interval. Curr Biol 25:2599-2609.

Leon MI, Shadlen MN (2003) Representation of Time by Neurons in the Posterior Parietal Cortex of the Macaque. Neuron 38:317-327.

Lynch JC, Hoover JE, Strick PL (1994) Input to the primate frontal eye field from the substantia nigra, superior colliculus, and dentate nucleus demonstrated by transneuronal transport. Exp Brain Res 100:181-186.

Mano N, Ito Y, Shibutani H (1991) Saccade-related Purkinje cells in the cerebellar hemispheres of the monkey. Exp Brain Res 84:465-470.

Maimon G, Assad JA (2006) A cognitive signal for the proactive timing of action in macaque LIP. Nat Neurosci 9:948-955.

Manto M, Bower JM, Conforto AB, Delgado-García JM, da Guarda SNF, Gerwig M, Habas C, Hagura N, Ivry RB, Mariën P, Molinari M, Naito E, Nowak D A, Oulad Ben Taib N, Pelisson D, Tesche CD, Tilikete C, Timmann D (2012) Consensus paper: roles of the cerebellum in motor control--the diversity of ideas on cerebellar involvement in movement. Cerebellum 11:457-487.

Merchant H, Zarco W, Perez O, Prado L, Bartolo R (2011) Measuring time with different neural chronometers during a synchronization-continuation task. Proc Natl Acad Sci 108:19784-19789. 
May PJ, Hartwich-Young R, Nelson J, Sparks DL, Porter JD (1990) Cerebellotectal pathways in the macaque: Implications for collicular generation of saccades. Neuroscience 36:305-324.

Ohmae S, Uematsu A, Tanaka M (2013) Temporally specific sensory signals for the detection of stimulus omission in the primate deep cerebellar nuclei. J Neurosci 33:15432-15441.

Ohmae S, Tanaka M (2016) Two different mechanisms for the detection of stimulus omission. Sci Rep 6:20615.

O'Reilly JX, Mesulam MM, Nobre AC (2008) The cerebellum predicts the timing of perceptual events. J Neurosci 28:2252-2260.

Oshio KI, Chiba A, Inase M (2008) Temporal filtering by prefrontal neurons in duration discrimination. Eur J Neurosci 28:2333-2343.

Ramnani N (2012) Frontal lobe and posterior parietal contributions to the cortico-cerebellar system. Cerebellum 11:366-383.

Ron S, Robinson DA (1973) Eye movements evoked by cerebellar stimulation in the alert monkey. J Neurophysiol 36:1004-1022.

Roth MJ, Synofzik M, Lindner A (2013) The cerebellum optimizes perceptual predictions about external sensory events. Curr Biol 23:930-935.

Schneider BA, Ghose GM (2012) Temporal production signals in parietal cortex. PLoS Biol 10:e1001413.

Strick PL, Dum RP, Fiez JA (2009) Cerebellum and nonmotor function. Annu Rev Neurosci 32:413-434.

Thach TW, Goodkin HP, Keating JG (1992) the Cerebellum and the Adaptive Coordination. Annu Rev Neurosci 15:403-442.

Thier P, Dicke PW, Haas R, Barash S (2000) Encoding of movement time by populations of cerebellar Purkinje cells. Nature 405:72-76.

Uematsu A, Tanaka M (2015) Effects of electrical microstimulation to the primate cerebellar dentate nucleus on the detection of stimulus omission in the missing oddball paradigm. $\mathrm{J}$ Physiol Sci 65 (Suppl):S287.

Yumoto N, Lu X, Henry TR, Miyachi S, Nambu A, Fukai T, Takada M (2011) A neural correlate of the processing of multi-second time intervals in primate prefrontal cortex. PLoS One 6:3-9. 


\section{Figure legends}

Figure 1. (A) Sequence of events in the missing oddball detection paradigm. Monkeys made a targeting saccade in response to the single omission of repetitive stimulus that surrounded the fixation point. Electrical stimulation was applied before the stimulus omission in a fraction of trials. (B) Sites of electrical stimulation in 3 monkeys reconstructed from either histological sections (monkeys H and I) or MRI (A). For some penetrations, stimulation sites are jittered horizontally only for presentation purpose. Labels indicate the posterior locations of coronal sections (in millimeters) relative to the inter-aural line.

Figure 2. Examples of neuronal activity and stimulation experiments. (A) Time courses of single neuronal activity recorded from a stimulation site. Vertical dashed lines indicate the timing of stimulus presentation. During the recording experiments, the omission occurred after a random 2000-4800 ms period from the first stimulus in the sequence. The regular repetitive stimulus terminated when the animals made a saccade to the target in response to the stimulus omission, but were presented as far as they maintained fixation. (B) Each pair of eye position traces compares the data from trials with and without electrical stimulation for different SOAs. Horizontal black bar indicates the timing of microstimulation (100 ms in duration, $100 \mu \mathrm{A}$ pulses at $333 \mathrm{~Hz}$ ).

Figure 3. Summary of stimulation effects on saccade latency. Each data point indicates each monkey and SOA, and compares the reaction time between trials with and without electrical stimulation. Error bar indicates \pm SD.

Figure 4. Effects of stimulation timing within each inter-stimulus interval. (A) Histograms indicate overall distributions of saccade latency relative to the stimulus omission for different stimulation timing (black horizontal bar). Data from 42 experiments in two monkeys were combined. (B) Means ( \pm SD) of median saccade latency for different stimulation timing. (C) Change in saccade latency $(\triangle \mathrm{RT})$ for each $100 \mathrm{~ms}$ advancement of stimulation timing. (D) Time course of population activity for neurons recorded in close vicinity of stimulation sites.

Figure 5. Rate of the occurrence of stimulation-evoked saccades for different timing of electrical stimulation in the sequence. In this series of experiments, the omission occurred either at the 13th or 14th stimulus timing. Electrical stimulation at the 8th timing was delivered in only 14 of the 20 experiments. 
Figure 6. (A) Three different temporally-specific signals during the missing oddball task. Signals predicting the timing of each next stimulus peak around the times of stimulus onset. Anticipatory signals for the occurrence of stimulus omission gradually increase overtime. Sensory stream with a short SOA induces sustained activity that ceases briefly in response to the stimulus omission. (B) Hypothetical diagram of signal flow during the task. Note that the cerebellum generates temporal prediction signals for each stimulus, while the signals related to the expectation of stimulus omission and sensory streaming are processed elsewhere. 

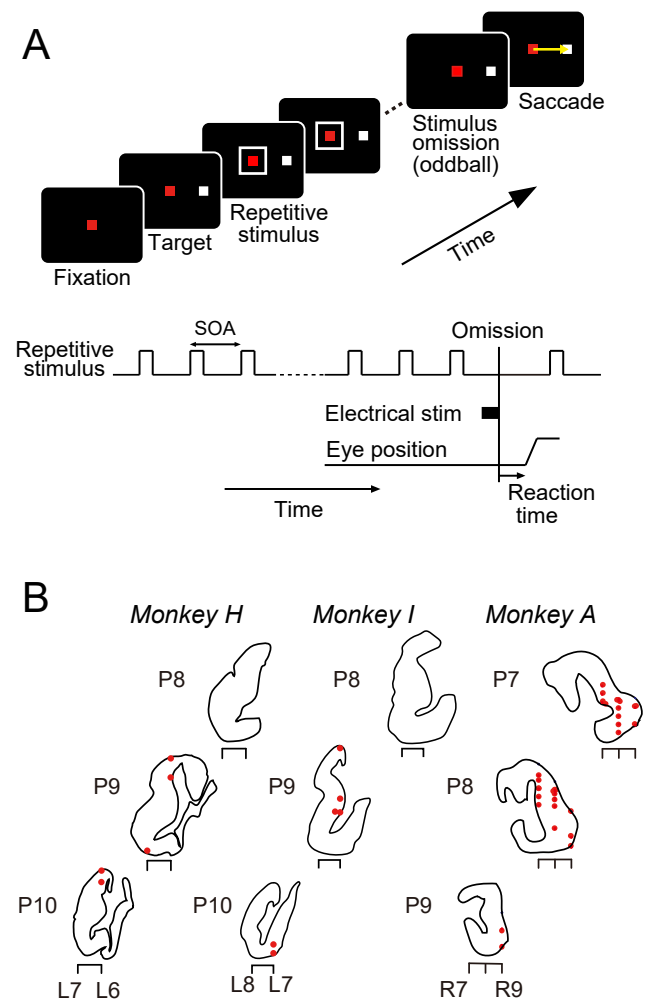

Fig.1 Uematsu et al. 


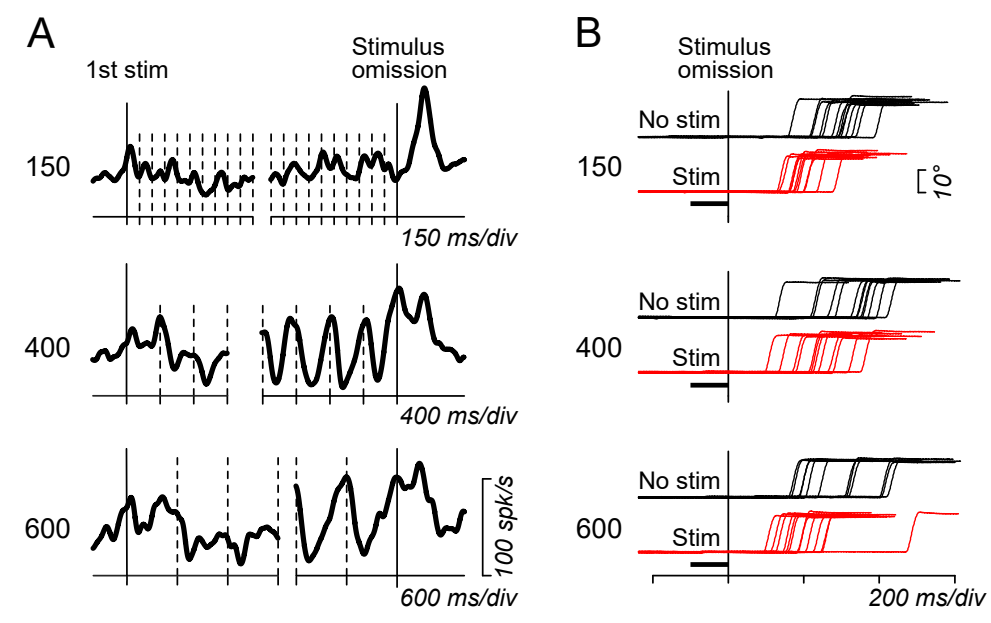

Fig. 2 Uematsu et al. 
Ipsi

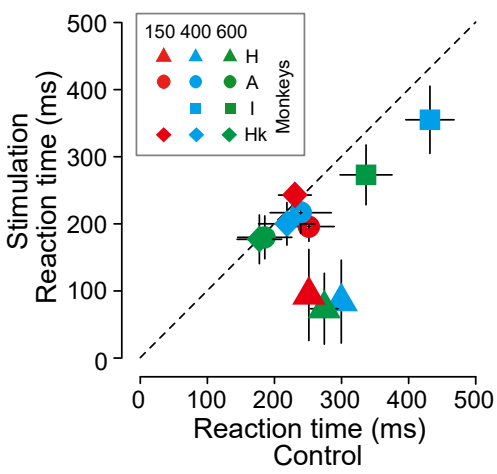

Contra

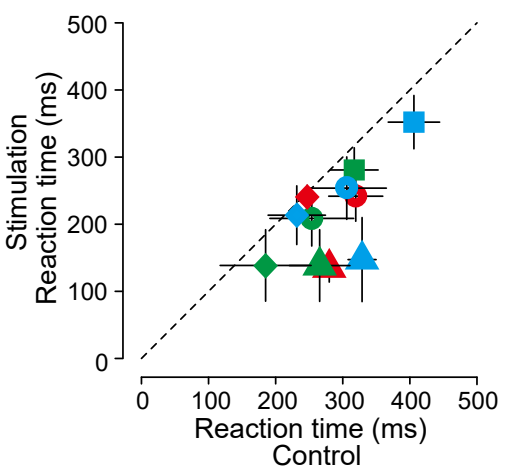

Fig. 3 Uematsu et al. 


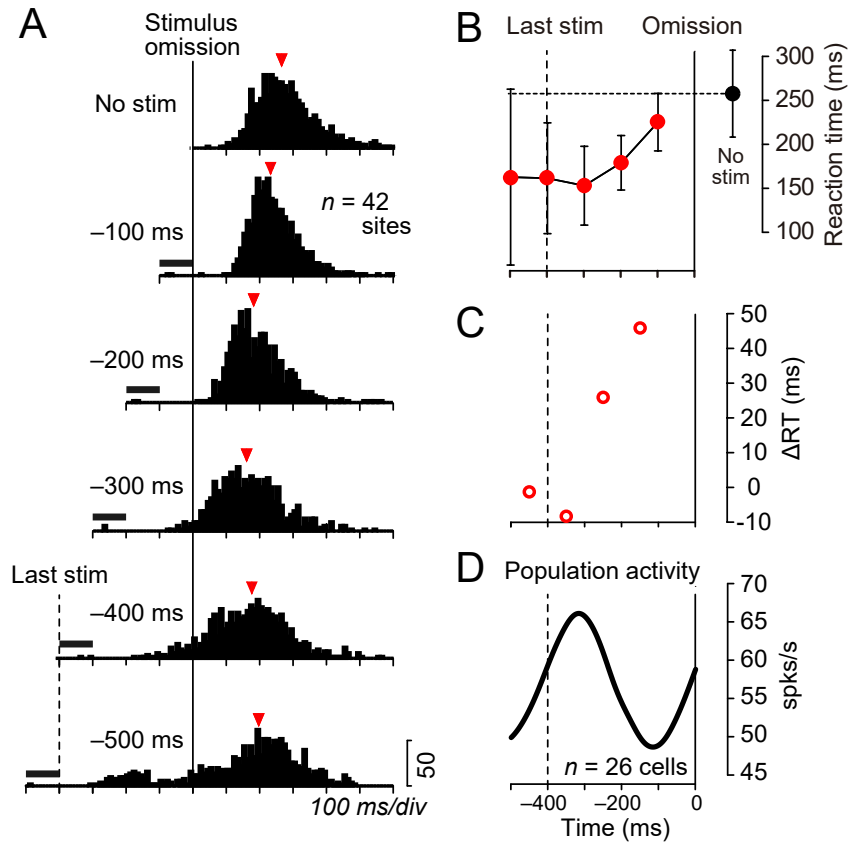

Fig. 4 Uematsu et al. 

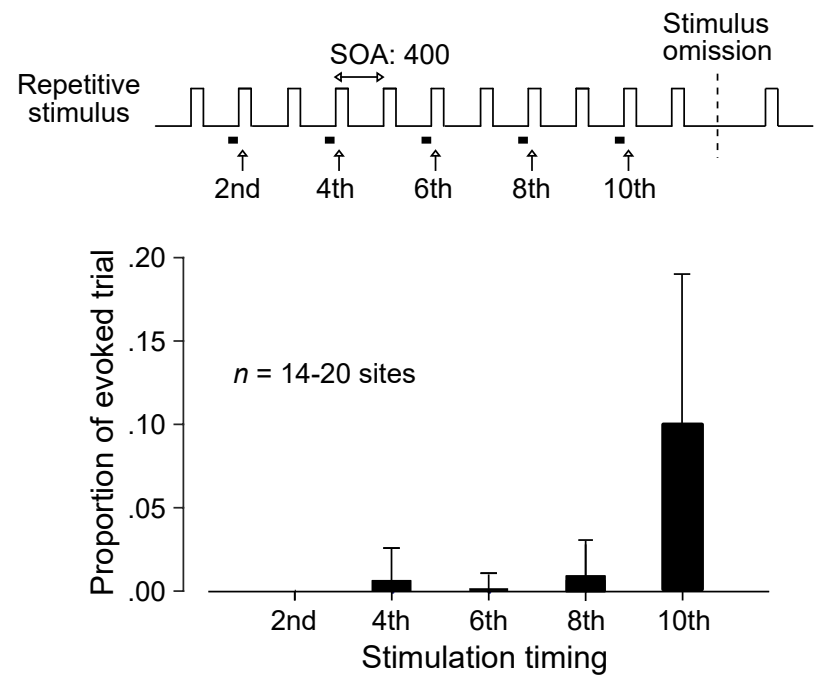

Fig. 5 Uematsu et al. 


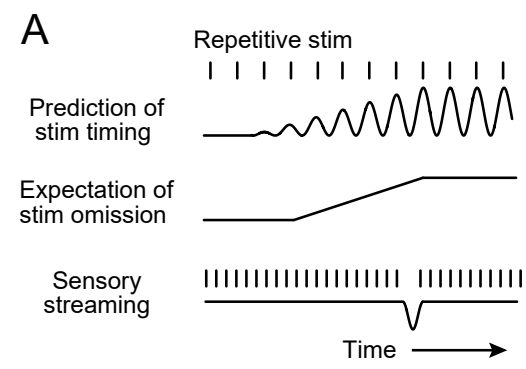

B

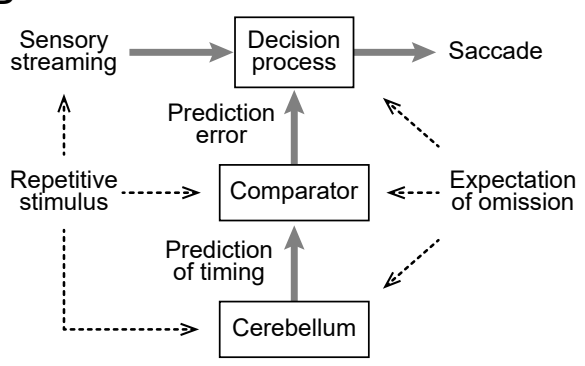

Fig. 6 Uematsu et al. 